\title{
EFFECTIVENESS OF PROPOLIS WITH ARTEMISININ COMBINATION THERAPY IN TREATING MICE INFECTED WITH PLASMODIUM BERGHEI
}

\author{
NURINDAH SALOKA TRISNANINGRUM ${ }^{1}$, HENDRI ASTUTY ${ }^{2 *}$ \\ ${ }^{1}$ Undergraduate Program, Faculty of Medicine, Universitas Indonesia, Jakarta, Indonesia. ${ }^{2}$ Department of Parasitology, Faculty of \\ Medicine, Universitas Indonesia, Jakarta, Indonesia. Email: astutiek@yahoo.com
}

Received: 30 September 2018, Revised: 30 September 2018, Accepted: 13 March 2019

\section{ABSTRACT}

Objective: This study aimed to ascertain the effectiveness of combination treatment with propolis and artemisinin-based combination therapy (ACT) in avoiding further resistance to ACT.

Methods: A total of 35 mice were injected with Plasmodium berghei and divided into six equal groups: No treatment (negative control), ACT alone (positive control), 75-mg propolis/kg body weight (BW), 150-mg propolis/kg BW, ACT with 75-mg propolis/kg BW, and ACT with 150-mg propolis/ kg BW. After 7 days of therapy, parasite density was calculated using a thin blood smear.

Results: Parasite density significantly declined after combination treatment with ACT and 150-mg propolis/kg BW.

Conclusion: Therapy with propolis alone showed no inhibitory effect on parasites, although its 150 -mg/kg-BW dose was effective as an ACT adjuvant malaria therapy in mice.

Keywords: Propolis, Malaria, Artemisinin-based combination therapy, Plasmodium berghei.

(C) 2019 The Authors. Published by Innovare Academic Sciences Pvt Ltd. This is an open access article under the CC BY license (http://creativecommons. org/licenses/by/4. 0/) DOI: http://dx.doi.org/10.22159/ijap.2019.v11s1.012

\section{INTRODUCTION}

Malaria is a common disease in many parts of the world. In 2015, the number of malaria cases reached 214 million worldwide, with 438,000 related deaths. Africa has the highest prevalence of malaria (88\%) followed by Southeast Asia (10\%) and the Eastern Mediterranean region (2\%). In 2014, 30,000,000 cases of high transmission malaria with $>1$ case per 1000 population and $36,500,000$ cases of the cases of low transmission malaria were reported in Indonesia. Despite this high number of cases, the global malaria burden is decreasing; the malaria incidence rate declined to $37 \%$ in 2015 from that in 2000 [1,2].

Several drugs are currently used to treat malaria infections. However, in some regions, Plasmodium has developed resistance to some drugs, making some therapies no longer effective. Resistance to artemisinin, sulfadoxine/pyrimethamine, mefloquine, chloroquine, and several other drugs is common in most parts of Africa and throughout most of Southeast Asia [3]. At present, artemisinin-based combination therapy (ACT) is used as the first-line treatment for malarial infections. Combination therapies are generally administered to improve the efficacy of resistant drugs.

In addition to using a combination of chemical drugs, antimalarial therapy is now developed using herbal ingredients mainly from the African region. Some plants, such as Parkia biglobosa, Tithonia diversifolia, and Nauclea latifolia, contain compounds with antimalarial activity in their roots, leaves, or stems. In addition, animals and animal products, including Malus domestica bees and bee products such as honey and propolis, have been investigated for antimalarial activity [4]. In a previous study, the decrease in parasitemia levels (PL) was studied in mice using a combination of peg earth and chloroquine; the results showed no significant decrease in parasitemia after therapy [5]. Syamsudin et al. showed that in Plasmodium-infected mice, propolis has an immunomodulatory effect, leading to enhanced immunity and improved survival [6].
This study aimed to develop antimalarial therapy using a combination of ACT and propolis, which is easily found in Indonesia. The combination of ACT with propolis is expected to reduce the PL in the study subjects, which can then be used to effectively treat malaria.

\section{METHODS}

This experimental study was conducted at the Animal Laboratory of Puslitbangkes, Jakarta, from April to December 2016. The study protocol had been approved by the ethics committee of the Faculty of Medicine, Universitas Indonesia. The study population included 35 healthy 3-4-month-old mice (Mus musculus; weight: 21-30 g) that were maintained at Puslitbangkes, Ministry of Health in cages containing five mice each. Mice that did not develop parasitemia and those that died during the study were excluded from the study. Mice were fed regularly with pellets and were exposed to natural light-dark cycles. The 35 mice were divided into seven treatment groups ( $\mathrm{n}=5$ per treatment):

a. Healthy (without any treatment): Five healthy mice that were not given parasites.

b. Control (+): Five mice injected with $0.2 \mathrm{ml}$ of parasites and given 49.3-mg ACT/kg body weight (BW).

c. Control (-): Five mice injected with $0.2 \mathrm{ml}$ of parasites without any treatment.

d. Treatment 1 (propolis 1): Five mice injected with $0.2 \mathrm{ml}$ of parasites and treated with 75-mg propolis/kg BW.

e. Treatment 2 (propolis 2): Five mice injected with $0.2 \mathrm{ml}$ of parasites and treated with 150-mg propolis/kg BW.

f. Treatment 3 (combo 1): Five mice injected with $0.2 \mathrm{ml}$ of parasites and treated with 49.3-mg ACT $/ \mathrm{kg} \mathrm{BW+75-mg} \mathrm{propolis/kg} \mathrm{BW.}$

g. Treatment 4 (combo 2): Five mice injected with $0.2 \mathrm{ml}$ of parasites and treated with 49.3-mg ACT/kg BW+150-mg propolis/kg BW.

Plasmodium berghei was obtained from the parasitology laboratory, Faculty of Medicine, Universitas Indonesia, and incubated in nitrogen tubes. The parasites were injected into the mice. Once the PL reached $1 \%, 0.2 \mathrm{ml}$ of parasites was injected intraperitoneally to each group. 
All groups of mice, other than the healthy control group, were injected with parasites on day 0 (D0) and the therapies were continued orally accordingly to group allotment from D1 to D3. The therapy was stopped on day 4 , but the blood sampling was performed until D7. On D8, the mice were euthanized by cervical dislocation. Mouse blood samples from D1 to D7 were taken from the tail veins. For this purpose, blood was dripped onto preparatory glass, and the tail was pressed against the wound until the bleeding stopped. The place of blood collection was the same each day.

Thin blood swabs were used for mouse blood preparations and were stained with $10 \%$ Giemsa dye. The PL was counted per 1000 erythrocytes. The parasitemia inhibition was calculated using the following formula:

\section{Controlled parasitemia- \\ Growthinhibition $=\frac{\text { Treated } \text { parasitemia }}{\text { Controlled parasitemia }} \times 100$}

The Kruskal-Wallis was used as a non-parametric test and MannWhitney U-tests was used as a post hoc test for comparisons. Data were analyzed using the SPSS v.20 program.

\section{RESULTS}

The average PLs of each group of mice were calculated (Fig. 1). Although the study was conducted up to D7, data of only up to D4 were used for analysis as it was consistent with the 4-day suppressive test theory. The PLs of the $C(-)$, propolis 1 , and propolis 2 groups were significantly different from those of the $\mathrm{C}(+)$, combo 1 , and combo 2 groups. The $\mathrm{C}$ (-) group experienced a continuous increase in PLs up to $48.4 \%$ on D4.

The propolis 1 and propolis 2 groups had similar patterns of changes in PLs (Table 1). In contrast to the C (-), propolis 1 and propolis 2 groups showed $3.1 \%$ and $3.4 \%$ decrease in PLs on D4. The C (+), combo 1 , and combo 2 groups exhibited low PLs $(<2.2 \%)$ until the end of therapy. An

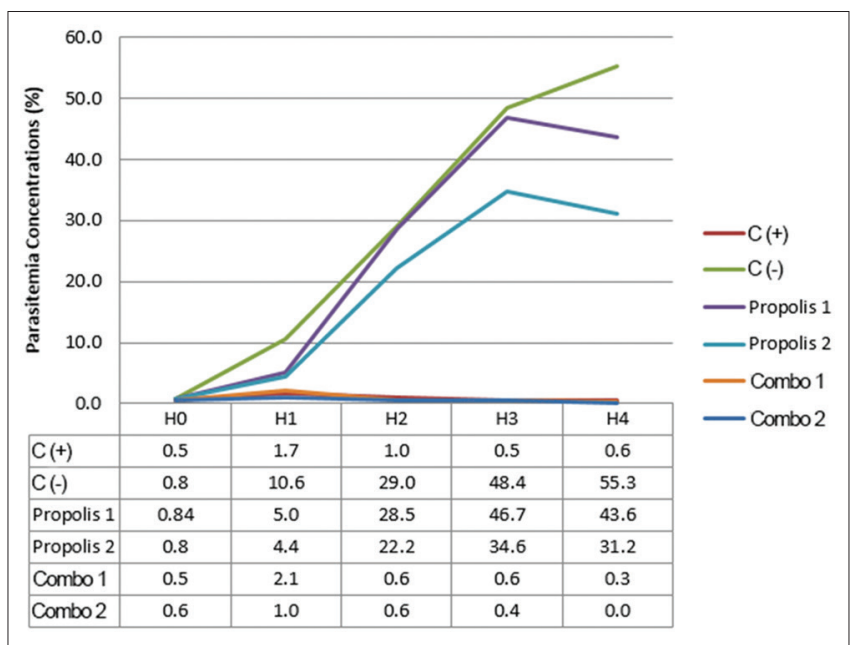

Fig. 1: Average parasitemia levels of DO-D4

Table 1: PLs and GI D0-D4 (\%)

\begin{tabular}{|c|c|c|c|c|c|c|c|c|c|}
\hline \multirow[t]{2}{*}{ Group } & \multirow{2}{*}{$\frac{\text { D0 }}{\text { PL }}$} & \multicolumn{2}{|l|}{ D1 } & \multicolumn{2}{|l|}{ D2 } & \multicolumn{2}{|l|}{ D3 } & \multicolumn{2}{|l|}{ D4 } \\
\hline & & PL & GI & PL & GI & PL & GI & PL & GI \\
\hline$C(+)$ & 0.5 & 1.7 & 83.77 & 1.0 & 96.69 & 0.5 & 99.01 & 0.6 & 98.88 \\
\hline$C(-)$ & 0.8 & 10.6 & - & 29.0 & - & 48.4 & - & 55.3 & - \\
\hline Propolis 1 & 0.84 & 5.0 & 52.64 & 28.5 & 1.59 & 46.7 & 3.51 & 43.6 & 7.96 \\
\hline Propolis 2 & 0.8 & 4.4 & 58.87 & 22.2 & 23.41 & 34.6 & 28.49 & 31.2 & 43.63 \\
\hline Combo 1 & 0.5 & 2.1 & 79.81 & 0.6 & 91.57 & 0.6 & 98.80 & 0.3 & 99.20 \\
\hline Combo 2 & 0.6 & 1.0 & 90.57 & 0.6 & 93.44 & 0.4 & 99.09 & 0.0 & 100.0 \\
\hline
\end{tabular}

PLs: Parasitemia levels, GI: Growth inhibition increase in PLs by $1.2 \%, 1.5 \%$, and $0.5 \%$ was observed in D1 in the C $(+)$, combo 1 , and combo 2 group, respectively.

The 4-day suppression test used the average data of PLs from D0 to D4 as a peak data. The C (-) group showed the highest PLs on D4 (55.3\%) followed by propolis 1 (43.6\%) and propolis 2 (31.2\%). By contrast, the $\mathrm{C}(+)$, combo 1 , and combo 2 groups showed very low PLs of $0.6 \%$, $0.3 \%$, and $0 \%$, respectively, on D 4 .

D0-D4 PLs were analyzed using SPSS v.20. The data were not significant $(\mathrm{p}<0.05)$ according to the non-parametric Kruskal-Wallis test (Table 2). The Mann-Whitney U-test was used to obtain the results are shown in Table 3.

According to the Mann-Whitney U-test, the $\mathrm{C}(+)$ and combo 1 groups, propolis 1 and propolis 2 groups, and combo 1 and combo 2 groups were not significantly different from each other.

The growth inhibition (GI) of the parasites was calculated by the previously mentioned formula (Fig. 2). The GI was calculated by the ratio $\mathrm{PL}$ of $\mathrm{C}(-)$ and to those of the treated groups to reveal how effectively the different treatments inhibited parasite growth up to D4.

In the propolis 1 and propolis 2 groups, a decrease in GI was observed from D1 (52.64\% and 58.87\% PLs, respectively) to D2 $1.59 \%$ and $23.41 \%$ PLs, respectively). GI increased from D3 to D4 (from $7.96 \%$ to $43.63 \%$ ). In D7, the C (+) group, GI increased up to $99.90 \%$. The combo 1 and combo 2 groups showed results similar to those observed in the C (+) group. From D0 to D3, the combo 2 group showed the highest increase in GI followed by the C (+) and combo 1 groups. On D4, the combo 1 group showed a GI of $99.20 \%$, which was higher than that of $98.88 \%$ in the $\mathrm{C}(+)$ group.

\section{DISCUSSION}

Propolis contains a variety of chemical compounds with various pharmacological effects including antioxidant, anti-inflammatory, antibacterial, anticancer, antifungal, and antiviral effects [7]. In accordance with the 4-day suppression test theory, we found the lowest PL on D4 in the groups receiving combination therapy of 150-mg propolis/kg BW and ACT, combination therapy of 75-mg propolis/kg BW and ACT, ACT alone, only 150-mg propolis/kg BW, only 75-mg propolis/kg BW, and no treatment. In addition, the combination of 150-mg propolis/kg BW and ACT showed the highest GI among all groups every day after D2.

GI was likely caused by the activity of flavonoids and hydroxycinnamic acid in propolis, which are powerful antioxidants that increase immunity $[8,9]$. Enhanced immunity likely improves the ability of ACT to damage the parasites circulating in the blood. Therefore, propolis can serve as an adjunctive therapy for improving the effectiveness of ACT as a standard malaria drug.

However, propolis therapy alone at doses of 75 and $150 \mathrm{mg} / \mathrm{kg} \mathrm{BW}$ did not show good results for use as a single malaria therapy. On D4, a decrease in PLs was observed in both single propolis therapy groups, whereas an increase was observed in the negative controls. This finding is consistent with that of a study by Syamsudin et al. who found a decrease in PL on D4 after treatment with propolis at doses of 25, 50,

Table 2: Kruskal-Wallis test results

\begin{tabular}{lllll}
\hline $\begin{array}{l}\text { Days of } \\
\text { Treatment }\end{array}$ & Dosage & n & Brinkmann index & p value \\
\hline H4-H0 & C $(+)$ & 5 & $14.70(-0.10-0.40)$ & $<0.001$ \\
& C $(-)$ & 5 & $33.00(49.00-69.10)$ & \\
& Propolis 1 & 5 & $27.00(39.90-45.10)$ & \\
& Propolis 2 & 5 & $24.00(6.60-44.65)$ & \\
& Combo 1 & 5 & $9.90(-0.60-0.00)$ & \\
& Combo 2 & 5 & $3.90(-0.80-0.10)$ & \\
& Total & 35 & & \\
\hline
\end{tabular}




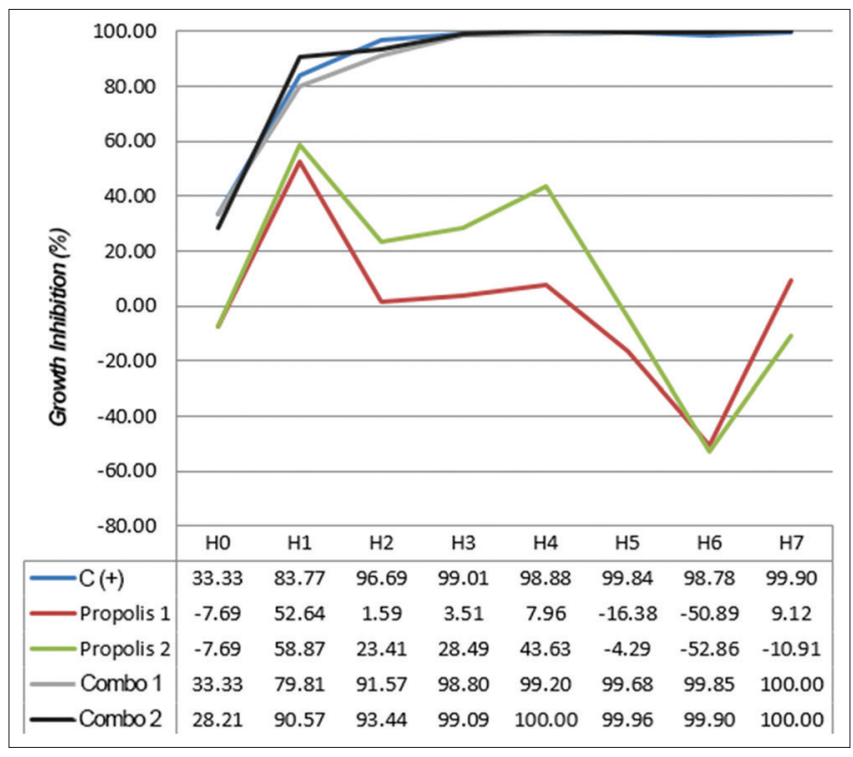

Fig. 2: Growth inhibition

Table 3: Mann-Whitney U-test results

\begin{tabular}{ll}
\hline Groups of Treatment & $\begin{array}{l}\text { Asymptotic } \\
\text { significance (two-tailed) }\end{array}$ \\
\hline C $(+)$ & $0.009^{*}$ \\
C $(-)$ & $0.009^{*}$ \\
Propolis 1 & $0.009^{*}$ \\
Propolis 2 & 0.147 \\
Combo 1 & $0.011^{*}$ \\
Combo 2 & \\
C (-) & $0.009^{*}$ \\
Propolis 1 & $0.009^{*}$ \\
Propolis 2 & $0.008^{*}$ \\
Combo 1 & $0.009^{*}$ \\
Combo 2 & \\
Propolis 1 & 0.116 \\
Propolis 2 & $0.008^{*}$ \\
Combo 1 & $0.009^{*}$ \\
Combo 2 & \\
Propolis 2 & $0.008^{*}$ \\
Combo 1 & $0.009^{*}$ \\
Combo 2 & \\
Combo 1 & $0.070^{*}$ \\
Combo 2 & \\
\hline * $<0.05$ &
\end{tabular}

and $100 \mathrm{mg} / \mathrm{kg} \mathrm{BW}$. However, from D1 to D3, no decrease was observed in PLs any of the single propolis therapy group administered [6].

During Plasmodium infection, mice develop antibodies according to their infection phase [7]. Syamsudin et al. showed that propolis treatment continuously increased the titer of IgG antibodies in mice from D1 to D4 [6]. On D4, a large increase was observed in GI compared with the increase on D3. However, this increase was not linear because GI on D1 was the highest. On D5-D7, single propolis treatment groups showed increase in parasite replication. The antiinflammatory effects of substances found in propolis, such as chrysin, gallangin, and m-coumaric acid, can suppress the immune response of mice resulting in increased parasite replication [9]. Differences between studies may be due to the varying levels of substances in propolis depending on the site of propolis production and the bees that produce it. The commercially obtained propolis is usually extracted using ethanol [10]. The use of commercial propolis from different geographical locations is likely to contain different compounds with pharmacological properties.

In general, combination therapy of propolis and ACT caused a higher decrease of PLs than the ACT alone. Thus, propolis at a dose of $150 \mathrm{mg} / \mathrm{kg}$ BW could be used as an additional therapy with ACT for malaria in mice. However, propolis administration alone could stimulate parasite replication. Further studies using human subjects are warranted to validate the use of this combination for treating human malaria cases.

\section{CONCLUSION}

The administration of propolis alone to experimental mice infected with P. berghei was not effective in reducing PLs compared with the combination of propolis and ACT. PLs after treatment with a combination of propolis and ACT tended to decrease, with GI higher than that by treatment with propolis or ACT alone.

\section{CONFLICTS OF INTEREST}

All authors declare that they have no conflicts of interest.

\section{REFERENCES}

1. World Health Organization. Number of Malaria Cases; 2014. Available from: https://www.who.int/gho/malaria/epidemic/cases/en/index1.html. [Last accessed 2018 Jul 07].

2. World Health Organization. Malaria; 2018. Available from: https:// www.who.int/news-room/fact-sheets/detail/malaria. [Last accessed 2018 Nov 19].

3. Bloland PB. Drug Resistance in Malaria. Switzerland: WHO Department of Communicable Disease Surveillance and Response; 2011.

4. Lawal B, Shittu OK, Kabiru AY, Jigam AA, Umar MB, Berinyuy EB, et al. Potential antimalarials from African natural products: A review. J Intercult Ethnopharmacol 2015;4:318-43.

5. Putri MA. Effectiveness of the Combination of Eurycoma longifolia with cq (chloroquin) as an Antimalarial Orally in Plasmodium bergheiInfected Mice. Undergraduate Thesis. Jakarta: Faculty of Medicine Universitas Indonesia; 2015.

6. Syamsudin S, Dewi RM, Kusmardi K. Immunomodulatory and in vivo antiplasmodial activities of propolis extracts. Am J Pharmacol Toxicol 2009;4:75-9

7. Stanistic DI, Fowkes FJ, Koinari M, Javati S, Lin E, Kiniboro B, et al. Acquisition of antibodies against Plasmodium falciparum merozoites and malaria immunity in young children and the influence of age, force of infection, and magnitude of response. Infect Immun 2015;83:646-60.

8. Li Y. Qinghaosu (artemisinin): Chemical and pharmacology. Acta Pharmacol Sin 2012;33:1141-6.

9. Toreti VC, Sato HH, Pastore GM, Park YK. Recent progress of propolis for its biological and chemical compositions and its botanical origin. Evid Based Complement Alternat Med 2013;2013:697390.

10. Jacob S, Nair AB. A simple practice guide for dose conversion between animals and human. J Basic Clin Pharm 2016;7:27-31. 